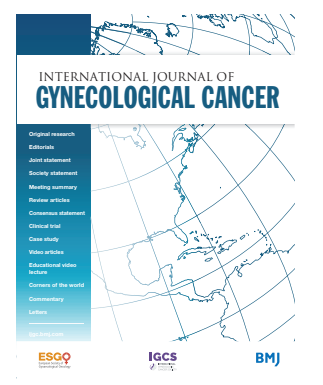

\title{
Across barriers: poly ADP-ribose polymerase inhibitors beyond progression in high grade serous ovarian cancer with brain metastases
}

\author{
Lawrence Kasherman \\ Medical Oncology and Hematology, Princess Margaret Hospital Cancer Centre, Toronto, Ontario, \\ Canada

\begin{abstract}
Ainhoa Madariaga Canada
Marjan Rouzbahman
Department of Laboratory Medicine and Pathobiology, University of Toronto, Toronto, Ontario, Canada \\ Medical Oncology and Hematology, Princess Margaret Hospital Cancer Centre, Toronto, Ontario,
Kieran Murphy
Joint Department of Medical Imaging, Princess Margaret Hospital Cancer Centre, Toronto, Ontario, Canada

David Shultz

Radiation Oncology, Princess Margaret Hospital Cancer Centre, Toronto, Ontario, Canada

Tracy Stockley

University Health Network, Toronto, Ontario, Canada

Amit M Oza

Medical Oncology and Hematology, Princess Margaret Hospital Cancer Centre, Toronto, Ontario, Canada

\section{Correspondence to Dr Amit M Oza, Medical Oncology and Hematology, Princess Margaret Hospital Cancer Centre, Toronto, ON M5G 2C9, Canada; amit.oza@ uhn.ca}

Accepted 24 August 2020 Published Online First 30 September 2020
A) Check for updates

(C) IGCS and ESGO 2021. № commercial re-use. See rights and permissions. Published by BMJ.

To cite: Kasherman $\mathrm{L}$, Madariaga A, Rouzbahman M, et al. Int J Gynecol Cancer 2021;31:139-143.

\section{CASE PRESENTATION}

A 47-year-old woman was referred to Princess Margaret Cancer Centre, Toronto, Canada, in November 2016, with abdominal pain, distension, and weight loss. Baseline serum Ca125 was 1615U/ $\mathrm{mL}$, and prior cytological analysis of ascitic fluid in October 2016 confirmed a diagnosis of high grade serous carcinoma, likely ovarian origin. Computed tomography showed omental caking with peritoneal carcinomatosis, ovarian masses, and pelvic, para-aortic, and ileocolic lymphadenopathy. Primary debulking surgery in December 2016 was attempted but aborted due to the extent of disease intraoperatively, and instead she underwent omentectomy to confirm the diagnosis.

\section{Dr Rouzbahman}

Infiltrative nodules were comprised of pleomorphic cells with high grade nuclear atypia and abundant mitoses, forming papillary architecture and slit-like spaces (Figure 1). Necrosis was readily identified. The immunohistochemical panel (Figure 2) shows positive staining for PAX-8, p16, WT-1, and overexpression of p53, and weakly patchy for estrogen and progesterone receptors. Taken together, the morphology and immunohistochemical pattern is consistent with high grade serous carcinoma, likely from tubal ovarian origin.

Subsequently, she received four cycles of neoadjuvant carboplatin and paclitaxel with good radiographic response. She then underwent total hysterectomy, bilateral salpingo-oophorectomy, and lysis of adhesions in March 2017, and was noted to have a complete response intraoperatively, with no residual disease visible at the end of surgery. The pathology specimen revealed no evidence of carcinoma. She underwent two further cycles of adjuvant chemotherapy, completed in May 2017. She also underwent germline testing using a multi-gene next generation sequencing panel (23 genes) and was found to harbor germline BRCA1 mutation c.1217delA, p.(Asn406Metfs*4), for which genetic counselors were consulted. Her family history was significant for two maternal aunts with ovarian cancer, and she had a background history of viral cardiomyopathy with mildly reduced left ventricular ejection fraction.

After a platinum free interval of 12 months, she presented with headaches and unsteady gait. Magnetic resonance imaging (MRI) of the brain demonstrated intracranial recurrence with right 


\section{Case study}

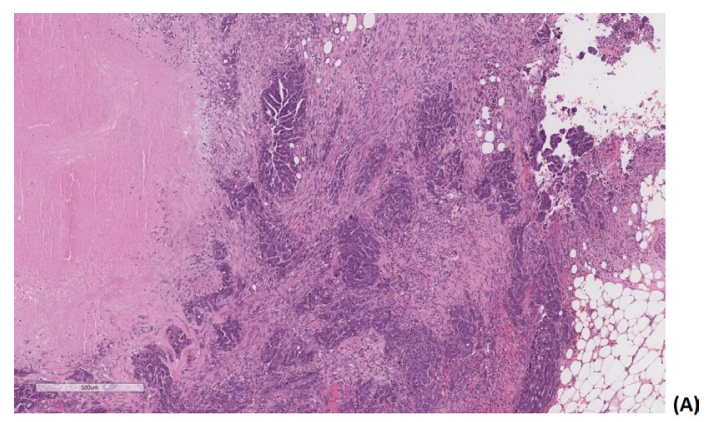

(A)

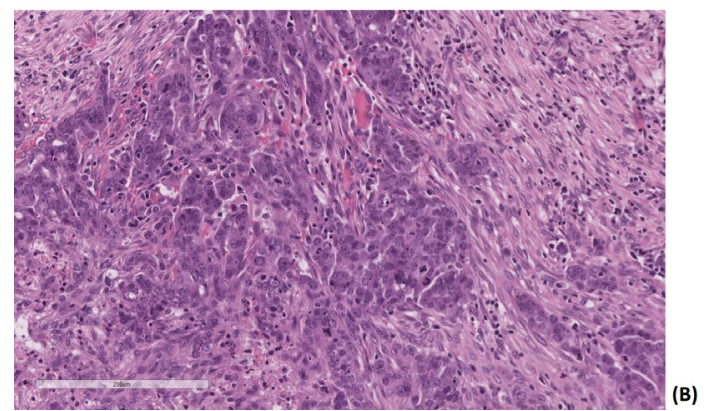

Figure 1 Hematoxylin and eosin staining. (A) Fibroadipose tissue ( $\times 50$ magnification) infiltrated by adenocarcinoma, demonstrating papillary architecture and slit-like spaces. Necrosis is present on the left side of the picture. (B) Higher $(\times 200)$ magnification of tumor, demonstrating tumor cells with marked nuclear atypia and numerous mitotic figures.

frontal lobe and left cerebellar metastases measuring $10 \mathrm{~mm}$ each. Ca125 was $22 \mathrm{kU} / \mathrm{L}$, and computed tomography restaging was unremarkable, aside from borderline enlarged axillary lymph nodes bilaterally.

\section{Dr Shultz: Can You Describe the Management Considerations for Intracranial Metastases in High Grade Serous Ovarian Cancer?}

Brain metastases are a rare manifestation of ovarian high grade serous carcinoma, occurring in $<2 \%$ of patients. ${ }^{1}$ Evidence regarding optimal management for brain metastases in ovarian high grade serous carcinoma remains limited due to its rarity. Like most carcinomas, treatment usually includes radiotherapy in addition to systemic therapy. Surgical management is often reserved for larger, symptomatic lesions, or when a pathologic specimen is required. ${ }^{2}$ Such multimodality approaches produce long term survival in selected cases. ${ }^{2}$ Stereotactic radiosurgery is the standard of care for patients with less than four lesions as it decreases the risk of cognitive toxicity compared with whole brain radiotherapy. ${ }^{3}$ When surgery is required, stereotactic radiosurgery targeting the surgical cavity similarly decreases the risk of toxicity compared with whole brain radiotherapy.

Our patient underwent stereotactic radiosurgery to a dose of $21 \mathrm{~Gy}$, targeting both intracranial lesions, followed by four cycles of platinum and gemcitabine, switching from carboplatin to cisplatin for the final two cycles due to hypersensitivity. She was commenced on maintenance olaparib, a poly ADP-ribose polymerase inhibitor, in November 2018. Furthermore, her archival surgical specimens were included in a molecular profiling study (NCT02906943) of 555 genes, where 15 variants were detected, including likely oncogenic variants in BRCA1, SMAD4, and TP53.
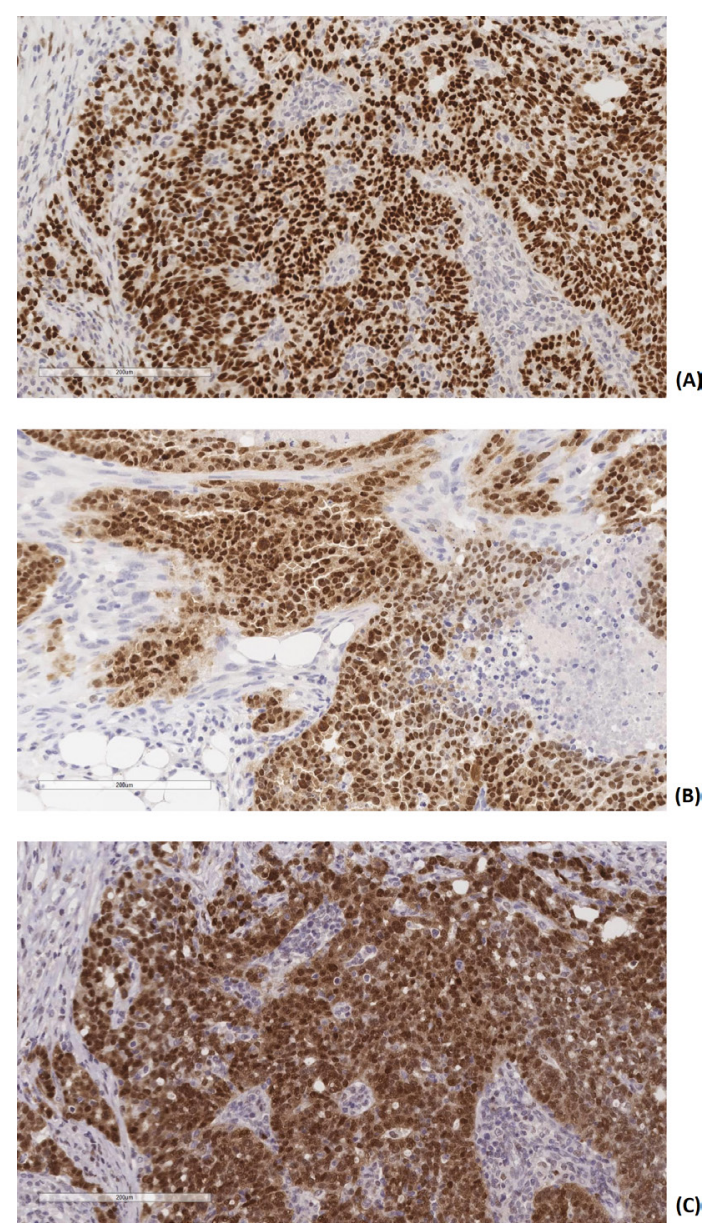

Figure 2 Immunohistochemical staining. (A) WT1: nuclear staining in the majority of tumor cells. (B) Pax8: nuclear staining in the majority of tumor cells. (C) p53: nuclear staining in more than $80 \%$ of tumor cells.

\section{Dr Rouzbahman and Dr Stockley: Could You Please Discuss Any Known Pathological Mechanisms Behind Development of Brain Metastases in Ovarian Cancer?}

To date, studies focusing on pathological markers predictive for development of brain metastases in ovarian high grade serous carcinoma have been very limited. Although the main route of spread in high grade serous carcinoma appears to be through peritoneal surfaces, occasionally hematogenous spread is encountered, usually to the liver or lung. Metastases to the central nervous system are quite uncommon and usually a late manifestation but rare reports of brain metastasis as the initial presentation of high grade serous carcinoma are present. ${ }^{2}$ Recent reviews on genomic alterations suggest that the presence of BRCA mutations or variants in other DNA repair pathways, such as ATM or CHEK2, increases the likelihood of developing brain metastases. ${ }^{14}$ Choi et al demonstrated on two patients that higher mutational burden and programmed cell death ligand-1 IHC expression was seen on brain metastatic tissue, but no single genomic alteration was attributable. ${ }^{5}$ Stasenko et al demonstrated that in 63 patients with BRCA mutations, overall survival was prolonged compared with BRCA wild-type patients but this finding became insignificant on controlling for extracranial disease, either due to the sensitivity of BRCA mutated cells to platinum or due to small sample size. ${ }^{6}$ Additionally, one study of 100 
gynecologic cancer patients also revealed that vascular endothelial growth factor-A, which is a pro-angiogenic factor targeted by bevacizumab, was expressed at slightly higher levels in metastatic brain tissue than primary tumor. ${ }^{7}$ It is possible that due to the increased number of available treatment options and subsequent prolonged survival times, that the number of patients presenting with brain metastases will increase due to tumor heterogeneity and isolation of resistant clones. ${ }^{8}$ Involving these patients in biomarker studies, particular where primary and metastatic tissue is available for analysis, remains of the utmost importance to achieve a better understanding behind the pathogenesis of brain metastasis.

\section{Dr 0za: Please Discuss the Use of Poly ADP-ribose Polymerase Inhibitors in Ovarian Cancer with Brain Metastases}

Poly ADP-ribose polymerase inhibitors used as maintenance therapy following response to platinum based chemotherapy in firstline and recurrent ovarian high grade serous carcinoma settings has become standard of care in those with BRCA mutations, with significant survival benefit and manageable toxicity rates, with no significant effects on quality of life. ${ }^{9}$ Systemic therapy in patients with brain metastases has historically posed a significant management challenge due to poor drug uptake across the blood-brain barrier, despite the hypothesis that the barrier is disrupted in the presence of intracranial metastases. In the preclinical setting, pharmacokinetic studies demonstrated antitumor activity in xenograft BRCA mutated tumors with therapeutic tumor growth inhibition to niraparib to a greater degree than olaparib. ${ }^{10}$ Mouse xenograft pharmacokinetic studies also showed accumulation and retention of rucaparib in tumor cells, but this was at a much lower level in brain tissue. ${ }^{11}$ One of the earliest observations of efficacy of poly $A D P$-ribose polymerase inhibitors in patients with brain metastases was reported by Professor Stan Kaye, who reported clinical activity and disease regression in a woman with endometrioid endometrial cancer and brain metastases in one of the earliest phase I patients being treated with olaparib. ${ }^{12}$ There have since been an increasing number of high grade serous carcinoma case reports documenting the clinical efficacy of this drug class in inducing prolonged intracranial tumor responses ${ }^{13-15}$; however, none of the published prospective poly ADP-ribose polymerase inhibitor studies in high grade serous carcinoma analyzed efficacy in patients with brain metastases, despite those with treated, stable brain metastases being eligible. Furthermore, those with symptomatic or untreated brain metastases were excluded from these studies. Currently, to my knowledge, there are no trials in progress assessing the activity of poly ADP-ribose polymerase inhibitors in ovarian cancer with brain metastases.

The activity of poly ADP-ribose polymerase inhibitors has also been assessed in other tumor sites. In breast cancer, where brain metastases are a more common occurrence, the phase III EMBRACA trial (A Study Evaluating Talazoparib (BMN 673), a PARPInhibitor, in Advanced and/or Metastatic Breast Cancer Patients With BRCAMutation) looking at talazoparib in BRCA mutated metastatic disease, detected a progression free survival benefit on subgroup analysis of 63 patients with brain metastases compared with chemotherapy alone (hazard ratio 0.32, 95\% confidence interval 0.15 to 0.68), implying clinical activity. ${ }^{16}$ In primary central nervous system tumors, the OPARATIC phase I trial demonstrated that after
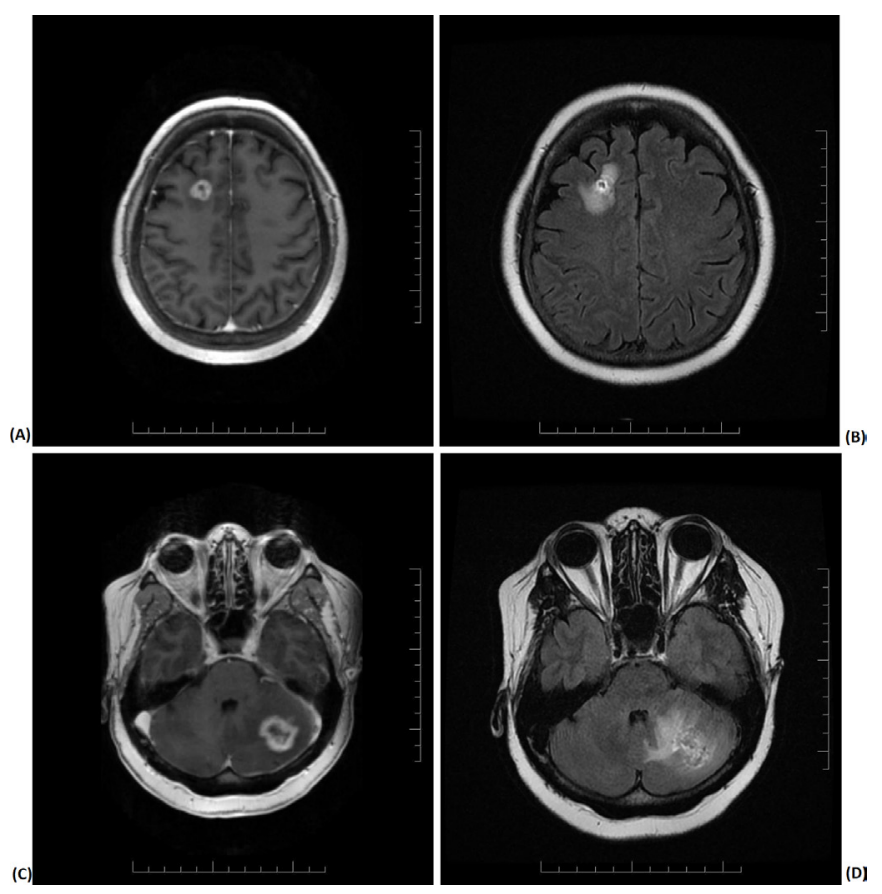

Figure 3 (A) Magnetic resonance imaging axial T1 weighted image showing the right frontal lobe ring enhancing lesion. (B) Fluid attenuated inversion recovery (FLAIR) image of the right frontal lobe lesion. (C) Axial T1 weighted image of the left cerebellar metastatic lesion. (D) Mild mass effect on cerebellar folia with surrounding FLAIR hyperintensity.

receiving olaparib with temozolomide, $100 \%$ of surgical resection specimens in 27 patients contained radiosensitizing concentrations of olaparib. ${ }^{17}$ Although data from these studies are limited and have small sample sizes, they provide encouraging signals of the potential clinical utility of poly ADP-ribose polymerase inhibitors in controlling intracranial malignancies.

In October 2019, both intracranial lesions demonstrated progression on surveillance brain MRI despite stable extracranial disease. Her brain metastases were targeted again with stereotactic radiosurgery, 21 Gy in three fractions, with a 3 week break in olaparib. In January 2020, the patient developed symptoms consistent with increased intracranial pressure, and surveillance brain MRI revealed increased vasogenic edema associated with her known metastases, requiring a several weeks course of dexamethasone. Due to repeated prolonged courses of steroids, she developed steroid induced diabetes mellitus. She also experienced a transient, acute rise in liver transaminases of unclear etiology requiring a 2 week break in olaparib. In June 2020, repeat imaging was obtained for surveillance, demonstrating no recurrent disease extracranially. An MRI was performed for surveillance, showing enlargement of a right frontal lobe lesion (Figure 3).

\section{Dr Murphy}

Axial T1 weighted images post-contrast demonstrated a right frontal lobe, subcortical white matter, and ring enhancing lesion with central necrosis (Figure 3A). Fluid attenuated inversion recovery imaging demonstrated surrounding edema but no significant mass effect (Figure 3B). In the posterior fossa there was a left cerebellar metastatic lesion demonstrating peripheral enhancement post-contrast with central necrosis (Figure $3 \mathrm{C}$ ). There was 


\section{Case study}

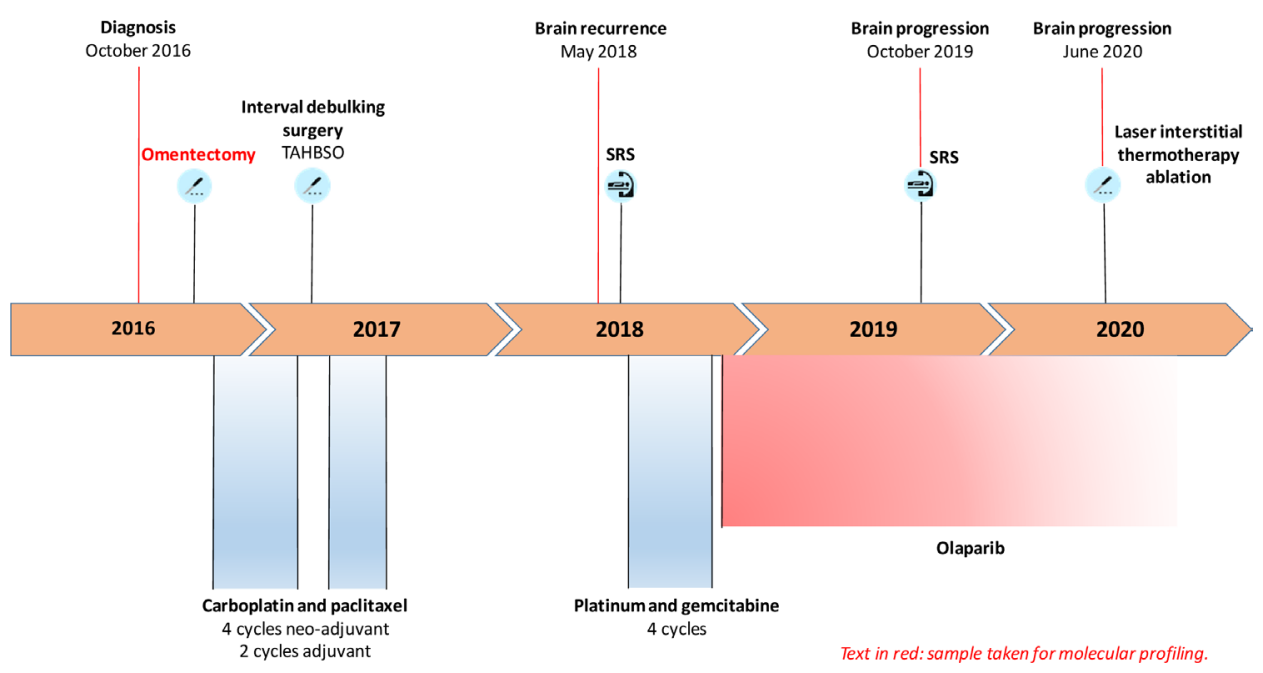

Figure 4 Summary of patient treatment history. Olaparib pending recommencement. SRS, stereotactic radiosurgery; TAHBSO, total abdominal hysterectomy and bilateral salpingo-oophorectomy.

surrounding fluid attenuated inversion recovery hyperintensity well seen on Figure 3D with mild mass effect on the cerebellar folia.

She was asymptomatic at the time. Following multidisciplinary discussion, she underwent craniotomy and laser interstitial thermotherapy ablation in late June 2020 and is pending recommencement of olaparib.

\section{Dr Oza: What is the Rationale for Continuing Poly ADP-ribose Polymerase Inhibition After Local Therapy in These Patients?} Despite the aforementioned reports, there is currently no literature describing whether poly ADP-ribose polymerase inhibitors should continue after local therapy for intracranial progression in the absence of extracranial disease. This case described asymptomatic intracranial progression without extracranial recurrence while on olaparib on multiple occasions, and following local therapy with either radiation or surgery, olaparib was continued. This practice of using targeted therapies beyond intracranial progression can be seen in other malignancies, such as non-small cell lung cancer, where epidermal growth factor receptor or anaplastic lymphoma kinase mutated tumors receive ongoing systemic therapy following local ablative treatments. ${ }^{18}$ Furthermore, consideration should be given to continuing poly ADP-ribose polymerase inhibitors beyond localized disease control in ovarian high grade serous carcinoma extracranial oligometastatic progression, given that progression in this context likely occurs within the context of clonal heterogeneity. Local treatment such as stereotactic body radiotherapy has been shown to achieve good local control, and several large poly ADP-ribose polymerase inhibitor studies have previously allowed continuation beyond progression. ${ }^{919}$ These clinical conundrums are highly individualized and require further evaluation in future studies.

One could argue that given the multiple instances of intracranial progression, that olaparib was failing to achieve disease control, which may be due to other factors, such as clonal heterogeneity. However, recommencement of chemotherapy is a less attractive option due to poor blood-brain barrier penetrance and risk of toxicity in the absence of extracranial disease. Another option would be to cease olaparib and continue on surveillance alone; however, given the presence of a BRCA mutation in the context of high grade serous carcinoma histology, there is concern that disease progression would occur rapidly, which could potentially be highly morbid if presenting as new intracranial metastases with vasogenic edema, or alternatively as widespread systemic disease. It is also possible that reversion mutations, or other molecular mechanisms pertaining to poly ADP-ribose polymerase inhibitor resistance, could be present given the duration of treatment. However, unfortunately, no biopsy was obtained at the time of craniotomy, and given the risk of extracranial disease progression, this would not alter management. A considered approach needs to be taken in managing patients in these situations, and transparent discussions should be had with each patient to ensure the optimal individualized management strategy.

\section{CLOSING SUMMARY}

This case describes a woman with ovarian high grade serous carcinoma diagnosed with brain metastases on platinum sensitive recurrence, for which following stereotactic radiosurgery and platinum based chemotherapy she was commenced on olaparib maintenance. She harbored a germline BRCA1 mutation. Due to the development of intracranial progression in the absence of extracranial disease, she underwent further radiation on a number of occasions and continued olaparib with manageable toxicity. A summary of her treatment history is displayed in Figure 4.

In conclusion, this case highlights the complex considerations associated with multimodal treatment in an ovarian high grade serous carcinoma subpopulation with intracranial metastases. Due to the rarity of brain metastases in this population, the evidence remains sparse and a number of questions have been raised surrounding the appropriateness of continuing poly ADP-ribose polymerase inhibitors in isolated intracranial progression. Standard practice guidelines for treatment of brain metastases in ovarian high grade serous carcinoma are yet to be clearly defined, and certainly the gynecologic oncology community will require increasing documentation of experience with poly ADP-ribose polymerase inhibitors in patients with brain metastases. With the increase in numbers of treatment options, identification of novel predictive markers and 
prolonged survival times, unusual manifestations of ovarian high grade serous carcinoma, such as brain metastases, are likely to become increasingly common. Prospective studies of diagnostic and treatment approaches will be necessary to understand the pathogenesis of brain metastasis and consequently identify optimal management strategies for these patients.

Acknowledgements The authors wish to acknowledge Katherine Karakasis and Dr Eric Chen for technical editing and review of the manuscript.

Contributors Presenters: LK and AM. Pathologist: MR. Radiologist: KM. Discussants: DS, TS, and AMO.

Funding The Ontario-wide Cancer TArgeted Nucleic Acid Evaluation (OCTANE) study was conducted with the support of the Ontario Institute for Cancer Research through funding provided by the Government of Ontario; grant Nos P.OCT.051 and P.A0.075.

Competing interests $\mathrm{AMO}$ is on the steering committee of GlaxoSmithKline, AstraZeneca, Clovis, Tesaro and Merck (uncompensated), and is PI on clinical trials for AstraZeneca, GlaxoSmithKline, and Clovis.

Patient consent for publication Obtained.

Provenance and peer review Commissioned; internally peer reviewed.

\section{REFERENCES}

1 Ratner E, Bala M, Louie-Gao M, et al. Brain metastases in primary ovarian cancer: real-world data. Ann Oncol 2018;29:viii338.

2 Marchetti C, Ferrandina G, Cormio G, et al. Brain metastases in patients with EOC: clinico-pathological and prognostic factors. A multicentric retrospective analysis from the MITO group (MITO 19). Gynecol Oncol 2016;143:532-8.

3 Kasper E, Ippen F, Wong E, et al. Stereotactic radiosurgery for brain metastasis from gynecological malignancies. Oncol Lett 2017;13:1525-8.

4 Balendran S, Liebmann-Reindl S, Berghoff AS, et al. Next-generation sequencing-based genomic profiling of brain metastases of primary ovarian cancer identifies high number of BRCA-mutations. $J$ Neurooncol 2017;133:469-76.

5 Choi YJ, Kim S-Y, Park H-C, et al. Integrative immunologic and genomic characterization of brain metastasis from ovarian/peritoneal cancer. Pathol Res Pract 2019;215:152404.
6 Stasenko M, Cybulska P, Feit N, et al. Brain metastasis in epithelial ovarian cancer by BRCA1/2 mutation status. Gynecol Oncol 2019;154:144-9.

7 Divine LM, Kizer NT, Hagemann AR, et al. Clinicopathologic characteristics and survival of patients with gynecologic malignancies metastatic to the brain. Gynecol Oncol 2016;142:76-82.

8 Yang SYC, Lheureux S, Karakasis K, et al. Landscape of genomic alterations in high-grade serous ovarian cancer from exceptional long- and short-term survivors. Genome Med 2018;10:81.

9 Madariaga A, Bowering V, Ahrari S, et al. Manage wisely: poly (ADPribose) polymerase inhibitor (PARPi) treatment and adverse events. Int J Gynecol Cancer 2020;30:903-15.

10 Mikule K, Wilcoxen K. Abstract B168: the PARP inhibitor, niraparib, crosses the blood brain barrier in rodents and is efficacious in a BRCA2-mutant intracranial tumor model. Mol Cancer Ther 2015;14:B168.

11 Murray J, Thomas $\mathrm{H}$, Berry $\mathrm{P}$, et al. Tumour cell retention of rucaparib, sustained PARP inhibition and efficacy of weekly as well as daily schedules. Br J Cancer 2014;110:1977-84.

12 Forster MD, Dedes KJ, Sandhu S, et al. Treatment with olaparib in a patient with PTEN-deficient endometrioid endometrial cancer. Nat Rev Clin Oncol 2011;8:302-6.

13 Gray S, Khor XY, Yiannakis D. Niraparib as maintenance therapy in a patient with ovarian cancer and brain metastases. BMJ Case Rep 2019;12:e230738.

14 Bangham M, Goldstein R, Walton H, et al. Olaparib treatment for BRCA-mutant ovarian cancer with leptomeningeal disease. Gynecol Oncol Rep 2016;18:22-4.

15 Sakamoto I, Hirotsu Y, Nakagomi H, et al. Durable response by olaparib for a Japanese patient with primary peritoneal cancer with multiple brain metastases: a case report. J Obstet Gynaecol Res 2019;45:743-7.

16 Litton JK, Rugo HS, Ettl J, et al. Talazoparib in patients with advanced breast cancer and a germline BRCA mutation. $N$ Engl $\mathrm{J}$ Med 2018;379:753-63.

17 Hanna C, Kurian KM, Williams K, et al. Pharmacokinetics, safety and tolerability of olaparib and temozolomide for recurrent glioblastoma: results of the phase I OPARATIC trial. Neuro Oncol 2020. doi:10.1093/neuonc/noaa104. [Epub ahead of print: 29 Apr 2020].

18 Kelly WJ, Shah NJ, Subramaniam DS. Management of brain metastases in epidermal growth factor receptor mutant non-smallcell lung cancer. Front Oncol 2018;8:208.

19 Lazzari R, Ronchi S, Gandini S, et al. Stereotactic body radiation therapy for oligometastatic ovarian cancer: a step toward a drug holiday. Int J Radiat Oncol Biol Phys 2018;101:650-60. 